\title{
New Opportunities in the Physics Landscape at CERN
}

\author{
Ewa Rondio* \\ CERN, Geneva, Switzerland \\ E-mail: Ewa.Rondio@cern.ch
}

The workshop "New Opportunities in the Physics Landscape at CERN" was held at CERN on 11-13 May 2009. The objective of the workshop was to explore new opportunities for physics at CERN using both existing accelerators and facilities and also accelerators foreseen for the upgrade of the LHC injection complex. The workshop was very well attended with a total of about 400 registered participants attending the three-day event with lively discussion periods during the formal sessions. The terms of reference of the workshop restricted discussion to experiments that could be done at CERN in the future and that could not be performed at other facilities. To this end, presentations on the status and future plans for facilities at other laboratories were scheduled. Moreover, the subject of neutrino physics was only treated in as much as its requirements for the future proton drivers at CERN. The Workshop, together with the additional scheduled events, will allow for the preparation of a CERN plan for the future non-LHC programme and also serve as an input to the world-wide coherent plan for non-LHC physics. We consider that the Workshop addressed adequately its aims that were put forth at the outset. In order to follow-up on any proposals, the SPSC and INTC Committees will review submitted proposals as per their normal procedures.

2009 KAON International Conference KAON09,

June 09 - 122009

Tsukuba, Japan

${ }^{*}$ Speaker. 
Detailed information concerning the workshop, including program, transparencies, session recording and summaries of the session prepared by convenors can be find on the web [1].

\section{Overview of the Ideas Discussed}

The short summaries of subjects discussed during the sessions is presented below. It is done following the workshop organization: session by session. The basis for what follows are the summaries prepared by session convenors.

\subsection{SPS - Deep Inelastic Scattering}

All abstracts submitted to this session focused on data taking with the COMPASS experiment and address open questions of the spin structure of the nucleon. The session started with an overview of the recent developments in Lattice-QCD and phenomenology important to unravelling the spin structure of the nucleon. The progress in lattice calculations should be especially mentioned, i.e. the first calculations on the orbital momenta of the $u$ and d quarks.

The measurement of exclusive reactions, i.e. deeply virtual Compton scattering, allow constrains of generalized parton distributions (GPDs). Proposed measurements will allow reaching smaller $\mathrm{x}$ than currently available to other fixed target experiments like HERMES or at JLab. The reach in Q2, a variable extremely important for the extraction of GPDs via evolution, can only be increased if the luminosity can be increased by a factor of five. In the short time scale Comapss proposes running with a polarised hydrogen target at $200 \mathrm{GeV} / \mathrm{c}$ beam energy: one year with longitudinal polarization to improve existing data at low $x$ and one year with transverse polarization, which would allow significant increase of the statistics for scattering on proton. Compass proposes also to measure Drell-Yan on a transversely polarised and unpolarised target.

An idea to improve the extraction of the unpolarised quark distributions by measuring with unpolarised hydrogen and deuterium targets and the COMPASS spectrometer the ratio of F2n/F2p to $0.1 \%$ accuracy was presented and discussed, with the conclusion that closer contact with Compass experiment is needed to define details of such measurement.

\subsection{SPS - Rare K-decays and CNGS}

The session consisted of one contribution on Rare K-decays (charged $K \rightarrow \pi \nu \bar{v}$ ) by experiment NA62 at the SPS, where a measurement with precision around 10\% of the SM prediction will be sensitive to loop effects coming from new physics at higher energy scales, and four contributions on the lepton sector, of which three combine frontline neutrino physics measurements with advancements in large Liquid Argon TPC technology. The proposed activities based on large liquid Argon detectors, are timely and important if Europe is to play a leading role in this future discovery program, even in the case that the actual experiments will not take place in Europe.

CERN has a long and successful track record in rare kaon decays and currently one of the frontline experiments under preparations and clearly this is a very exciting programme at the international frontline, and as such merits continued support. NA62 is preparing to measure the charged mode, while E14 at J-PARC propose to measure the neutral one. The NA62 program was presented at this workshop in a dedicated talk by M. Pape. 
For neutrino physics the proposal was presented to revive the PS neutrino beam and use two identical in terms of design LAr detectors at $127 \mathrm{~m}$ and $550 \mathrm{~m}$ from the target, both inside CERN. The detectors are based on ICARUS with significant improvements and simplifications from the lessons learned from it, offering 10 and 500 tonnes of fiducial mass respectively. With $2.5 \times 10^{20}$ POTs of neutrino and the same of antineutrino running the experiment will have thousands of fully reconstructed neutrino events and will address conclusively the LSND issue.

Recently the case for the existence of heavy sterile neutrinos was postulated again, in the vMSM model where three sterile $\mathrm{N}$ are assumed with masses in the $\mathrm{keV}-\mathrm{GeV}$ region. Using $10^{21}$ POT of $19 \mathrm{GeV}$ protons, one could improve the limits for $\mathrm{N}$ with $\mathrm{m}<450 \mathrm{MeV}$ by a factor of up to 20 compared to PS191. Using a TeV energy proton beam from a new source (SPS+) one could do a beam dump experiment extending the $\mathrm{N}$ mass range up to the $\mathrm{B}$ mass.

\subsection{SPS - Hadrons and Ions}

Hadron physics aims at a fundamental understanding of all bound and resonant systems and their interactions that are subject to the strong force. In the macroscopic world, QCD manifests itself through the underlying symmetries, chiral symmetry for the light (up, down, strange) and heavy quark symmetry for the heavy quarks. One key problem of QCD is to understand its spectrum. Most observed states could be understood as conventional mesons or baryons. However, the theory also predicts glueballs and also more exotic forms of matter, like tetra- or penta-quarks or hybrids. There are some indications for the existence of such states in the light-quark sector, but the overall picture remains puzzling.

The fixed target baryon spectroscopy was represented by proposals for search for doubly charmed baryons and essential tests of chiral perturbation theory, particularly by measuring the pion (and kaon) polarisabilities and the chiral anomaly amplitude. Scattering high-relativistic charged pions in the Coulomb field of nuclei denoted the Primakoff effect, accesses (quasi-real) $\gamma \pi$ reactions with different final states. The measurements require pion and kaon beams and a spectrometer with high rate capability and excellent electromagnetic calorimetry, which are available with SPS-M2 beam line and the COMPASS spectrometer.

Scientific plans of the DIRAC experiment, which main task is to check precise predictions of low-energy QCD using $\pi \pi$ and $\pi K$ atoms, is to obtain experimentally a value of the Lamb shift DeltaE $(2 s-2 p)$ in this atoms. New possibilities to check the predictions of the low-energy QCD would be available after the installation of the DIRAC setup on the $450 \mathrm{GeV}$ SPS proton beam.

Large experimental and theoretical efforts worldwide are devoted to the investigation of strongly interacting matter under extreme conditions. The goal of these activities is to explore, in the laboratory, the properties of such matter as function of temperature and density. The NA61/SHINE experiment at the CERN SPS aims to discover the critical point of strongly interacting matter and study properties of the onset of deconfinement. These goals will be reached by measurements of hadron production properties in nucleus-nucleus, proton-proton and proton-lead interactions as a function of collision energy and size of the colliding nuclei.

Also other signals of phase transition can be studied. Charm production is a good probe of hot QCD matter because due to the high mass of the charm quarks they are expected to be dominantly from hard scatterings between initial state partons. Measurements of yields and spectra will provide unique new insight in the nature of the hot QCD matter at SPS. So far, no direct charm measurement 
has been performed at SPS. Another key observable, J/psi suppression, exhibits a threshold-like behavior at top SPS energy, when studied as a function of the centrality of the collision. The extension of its systematic study towards lower energy could reveal detailed information on the nature of the observed threshold behavior, and allow a more direct connection with the underlying physics mechanism.

\subsection{PS and Non-accelerator Experiments}

The abstracts describing non-accelerator experiments all address burning questions in the SM with an overlap with Astroparticle Physics. These abstracts involved dark matter searches, axion searches, a search for proton decay and Astroparticle Physics searches for e.g. energetic neutrinos. Some new proposals particularly in Astroparticle Physics request a symbolic connection with CERN in a similar manner to the existing recognised experiments. These are the use of CERN as a centre for meetings, use of CERN as an executive financial institution, use of test beams and as a centre for R and D and assembly and testing of detectors. An interesting suggestion, which came up in the discussion, is that it may be advantageous for CERN to set up an Astroparticle Physics department similar to the one at FNAL. This would act as an umbrella for the experiments with astrophysical connections in both the new proposals and existing recognised experiments.

Two further abstracts each required access to particle beams. One abstract, of outstanding merit, proposed a search for the electric dipole moment (EDM) of the proton and deuteron. This would improve the sensitivity over the current neutron EDM limit by 3-4 orders of magnitude. The experiment has been proposed at BNL. However, the proposers believe that it would be done much faster at CERN. The second abstract was the CLOUD experiment which described its plans for the coming years. While few people believe that there is a strong link between global warming and cosmic rays, nevertheless it is interesting from the point of view of both meteorology and climatology to establish whether or not ionization from cosmic rays plays a part in cloud formation.

\subsection{ISOLDE}

ISOLDE is a radioactive ion beam facility using the ISOL production method. The radioactive species are produced by protons at $1 \mathrm{GeV}$ or $1.4 \mathrm{GeV}$ issued by the PS booster which impinge on various targets and induce spallation, fragmentation and fission reactions. The ISOLDE user community has funded and implemented several unique experimental set ups. To ensure that these investigations will continue to be at the forefront of radioactive beam science, improvements will be in three areas: energy, intensity and quality. These upgrades are referred to as the HIE ISOLDE project. Two phases of the project were presented and discussed.

The ISOLDE community submitted 31 abstract to the workshop. The subjects addressed include the structure of light neutron-rich nuclei and the testing of ab-initio and cluster calculations, the study of extended neutron distributions such as neutron halos and neutron skins, the mapping of the nuclear shells far from stability, the study of pairing in both $\mathrm{T}=1$ and $\mathrm{T}=0$ channels, the study of octupole shapes and the search for atomic EDMs enhanced by octupole deformation of the nucleus.

All three European ISOL facilities (HIE-ISOLDE, SPIRAL2 and SPES) as well as FAIR will be necessary to cover the broad physics programme laid out for radioactive beams in the next decade and the needs of the community. This has been recognized in the long range plan of Nu- 
PECC, in which HIE-ISOLDE is cited as an essential "intermediate generation" facility on the path to EURISOL.

\section{6 n-ToF}

The n-TOF, CERN's neutron time-of-flight facility based on the spallation pulsed white neutron source, has become a unique world-leading facility for the measurement of nuclear cross sections of neutron induced reactions, particularly for radioactive or rare material samples. With the upgrades proposed by the collaboration, particularly the construction of a second short path experimental area and eventually the utilization of the SPL/PS2 beams, the facility will surpass during many years all other existing time-of-flight facilities for this type of measurements. After an initial period of data taking from 2000-2004, the facility has been refurbished and upgraded to today's security standards at CERN and data taking has just been started. The performed measurements respond to the need for accurate nuclear data for nuclear astrophysics and in particular for stellar evolution and nucleosynthesis calculations.

The inclusion of n-TOF data in the most recent model calculations and evaluations for international nuclear data libraries and in the astrophysical libraries illustrates the claimed impact. For successful development several improvements and extensions of the facilities were considered. The construction of a class A laboratory in the experimental area will provide a much wider range of samples to be measured without compromising the safety of the experiment. A second experimental area with short flight path at about $20 \mathrm{~m}$ would increase the neutron flux by a factor of 100 . On the longer term, the integration of n-TOF within SPL or PS2 could provide a further increase in the brightness of the neutron source. The n-TOF facility needs an intense pulsed proton beam.

\subsection{Antiproton Decelerator (AD)}

Access to low energy antiprotons $(\bar{p})$ offers excellent and unique opportunities to study the properties of fundamental forces and of symmetries in nature. Experiments with $\bar{p}$ can substantially contribute to our knowledge of atomic, nuclear and particle physics, in a complementary way to that to be acquired at the highest energy accelerators such as LHC. Concrete motivations arise in a number of theoretical approaches extending the SM and GR to include a consistent unified description of three cornerstones of physics: Lorentz symmetry, quantum mechanics, and gravity. The Standard-Model Extension(SME) governs a large set of the emerging effects relevant for lowenergy antimatter experiments.

At present, four experiments take data at the CERN AD. The three experiments which are the heaviest users of beam time are alphabetically ALPHA, ASACUSA and ATRAP. The ACE experiment addresses the issue of $\bar{p}$ in cancer therapy. The main goal of the proposed and recently approved AEGIS experiment is the first ever measurement of the gravitational interaction of $\bar{H}$ to $1 \%$. The expectation is that $\bar{H}$ spectroscopy will provide comparisons of $\bar{H}$ and $\mathrm{H}$ at much higher precision (presently $\approx 10^{-9}$ ). Formation of beams of $\bar{H}$ in the ground state is an alternative approach to trapping and also allows to study the gravitational properties of $\bar{H}$. The new ideas presented at this workshop include: a measurement of $\bar{H}$ acceleration in the gravity field of Earth, study of spin dependence in $p \bar{p}$ interactions, double strange production with $\bar{p}$ and antiprotonic atoms $\mathrm{X}$-ray studies for low $\mathrm{Z}$ elements. The newly proposed experiments would further reduce the availability of $\bar{p} \tilde{O} s$. 
The efficiency of the experiments would be much improved and the productivity and the availability of the unique user facility $\mathrm{AD}$ at CERN with its great scientific potential would be greatly enhanced if a further deceleration and cooling storage ring would be installed between the AD and the experiments. Such a ring could be the suggested ELENA ring. It is envisaged that ELENA will increase the phase space density at $100 \mathrm{keV}$ by one to two orders of magnitude, depending whether the experiments are already using the RFQD or not, respectively. This would raise the efficiency of the / program at CERN by a very large factor. The construction of a rather small machine for this purpose is feasible.

\subsection{Test Beams and Irradiation Facilities}

CERN provides unique facilities for test beams and irradiations with excellent technical support. There will be other possibilities for tests with high-energy particle test beams in the coming decades. The number of users of these facilities increases (eg. 22 in 2001 and 29 in 2009) and it is expected to continue, as tests of the LHC detectors will continue at least until 2012. More communities (sLHC, future detectors for a linear collider, heavy ion and neutrino physics) will increase their R\&D program with the need for test beams even longer than 2020.

The communities stress the need for permanent installation of their equipment within a test beam zone and see the need for additional infrastructure (magnets, beam instrumentation). The users ask for an opportunity for a regular forum to express their wishes, synchronise requests and exchange information and technology.

The test beams provide an excellent opportunity for education: for students they are very often the only possibility to get hands-on experience with detectors and to learn about experimental techniques. The technical support at CERN for the users was described as superb and is vital for successful tests also in the future. The discussion concentrated on the tests beams for noncollider, collider and future irradiation facilities at the CERN test beams. For the communities interested in heavy ions, additional possibilities for test (and physics) beams with different ion species were proposed. The test beam needs for neutrino detector R\&D will start to increase from 2010 onwards. For future neutrino experiments, high power proton beams need to be used to provide the required neutrino flux. New targets for neutrino factories need to be developed, different possibilities (solid targets, liquid targets) need to be studied. With the increasing number of users from the collider experiments it can be expected that it will be required to have a larger number of permanent installations in the SPS North Area.

\subsection{Possible Future Developments}

The session received contributions on a few rather different topics, going from the search of new hypothetical particles or studies of QCD in various regimes, to studies of plasma physics in the LHC beam dumps or R\&D on new acceleration techniques. They were reviewed in three categories: (1) experiments that make use of the existing beams, and whose running does not directly impact the standard LHC operations (2) experiments that directly impact the default LHC running operations, or require new infrastructure for the beam line and/or experimental halls (3) new accelerator technologies.

A simple extension of the SM with one light singlet fermion per family could provide all the necessary ingredients to explain neutrino masses, the baryon asymmetry. Meson decays like 
$K \rightarrow \mu N, D \rightarrow \mu N$ and $B \rightarrow \mu N$, followed by the downstream decay of $N \rightarrow \mu^{+} \mu \nu$, provide experimental signatures that allow to probe the $\mathrm{N}$ mass up to a few $\mathrm{GeV}$. The use of the SPS beam allows the exploration of a mass range larger than what achievable at any other planned facility.

Proposals using modified configuration of the LHC beams include: 1). mono-energetic electron and photon beams which can be obtained by attaching electrons to heavy nuclei. This could allow ep and eA collisions. In the ep case, the CM energy is $205 \mathrm{GeV}$ and a luminosity of about $10^{29}$ can be achieved. The eA collisions at these energies would be a novelty, and unique. 2). The deployment of a ribbon target around the main orbit of the LHC beam, near the interaction point of the ALICE detector, to trigger fixed-target-like interactions induced by the beam halo. However, there is no obvious advantage of using the LHC in a fixed-target mode over an energy scan at RHIC. 3). Measurement of magnetic moments of charmed baryons by extracting and directing LHC beams towards a fixed target, to produce highly boosted charmed baryons, whose magnetic moments are measurable using crystal channeling.

The new idea in beam acceleration is to use existing high-energy proton bunches to drive a plasma wakefield. A key element in realizing PDPWA is the production of a very short proton bunch. A proof-of-principle experiment based on PDPWA is then proposed for consideration as a future CERN project.

\subsection{Other Facilities}

This session had different character, it was an overview of the situation and plans in other labs. The reason for including this was to allow discussion of the future plans in the word context.

\subsection{New Proton Drivers at CERN}

Coherence between accelerators at CERN, which are currently being realized and planned for the future, should be co-ordinated with those being built elsewhere. For example, co-ordination between HIE-ISOLDE, SPIRAL2, FAIR and EURISOL should be strengthened and also that between the AD and FLAIR. Elsewhere, a study of plans at FNAL, BNL, KEK/J-PARC, GSI, LBL and JINR should be taken into account and common R\&D efforts should be sought where appropriate.

It was announced at the workshop that the new LHC injection chain up to and including PS2 will be available in 2020. In the current design, the injection chain is expected to be used only $25 \%$ of the time for the LHC itself.

\section{Summary}

A rich programme of research at the SPS and PS was identified for the next five years in the physics of heavy-ions, proton structure, antiprotons, $\mathrm{K}$ rare decays and neutrinos. The planned upgrades of the LHC injector chain should be designed taking into account also the longer-term requirements of the CERN fixed-target programme, particularly the need of beam intensities. The workshop showed relatively little interest on physics experiments with potential future extracted beams from the LHC and with an experimental area at the future PS2. 
Several issues were raised, which need to be followed-up and discussed. The main points are: the fixed-target ion programme, aims and focus of the proton structure programme, AD consolidation/upgrades including construction of ELENA ring, test beams for detector and accelerator R\&D and an enhanced role of CERN in astroparticle physics could exploit the connections between dark matter, proton decay, neutrinos and astroparticles.

Both ISOLDE and nTOF are unique and world-leading facilities and the planned upgrades for the new experimental area of nTOF and HIE-ISOLDE as the new higher energy and intensity facility - will keep these facilities at the forefront of their respective research areas. The strategy for upgrading the nTOF depends very much on the availability of EU programmes.

The follow up of the workshop will be preparation of letters of intent and proposal which should be evaluated by the scientific committees.

\section{References}

[1] web side of the workshop: http://indico.cern.ch/conferenceModification.py?confId=51128 